\title{
Subnationale Mobilisierung in den neuen EU-Mitgliedstaaten: Governance-Leitbilder subnationaler Verwaltungseliten in Polen und Ungarn*
}

\author{
Diana Pitschel/Philipp Studinger / Michael W. Bauer
}

\section{Einleitung}

Seit der Aufnahme der Beitrittsverhandlungen mit den Staaten der "LuxemburgGruppe" 1 sind mittlerweile mehr als zehn Jahre vergangen. Der Beitritt Polens, der Tschechischen Republik, der Slowakei, Ungarns, Sloweniens, Zyperns, Maltas und der drei baltischen Staaten Estland, Lettland und Litauen zur Europäischen Union (EU) liegt fast vier Jahre zurück. Wie „Europa" und die sich durch die Mitgliedschaft ergebenden realpolitischen Veränderungen in den neuen Mitgliedstaaten angekommen sind, ist in der politikwissenschaftlichen Forschung bislang hauptsächlich unter dem Stichwort der „Europäisierung" mit einem einseitigen Fokus auf die nationalstaatliche Ebene untersucht worden. Bedenkt man jedoch, dass insbesondere in der Integrations- und der Governance-Forschung gerade der subnationalen Ebene eine zentrale Rolle im europäischen Politikprozess zugeschrieben wird, stellt sich verstärkt die Frage, wie sich die Beziehungen zwischen den subnationalen Ebenen der neuen Mitgliedstaaten und der EU entwickeln bzw. entwickelt haben. Es scheint daher Zeit für eine erste Bestandsaufnahme, wie sich denn insbesondere die subnationalen Verwaltungseliten mit der neuen Realität der Politikgestaltung im europäischen Mehrebenensystem arrangieren.

Im westeuropäischen Kontext, d. h. in den alten EU-Mitgliedstaaten, wird diese Diskussion seit geraumer Zeit unter dem Stichwort „subnationale Mobilisierung“ geführt. Unter subnationaler Mobilisierung versteht man gemeinhin die Intensivierung des Dialoges zwischen der subnationalen Ebene und der EU und ein verstärktes Engagement subnationaler Akteure in der europäischen Politikgestaltung. ${ }^{2}$ Es geht also dabei keineswegs um die einseitige Orientierung der Regionen in Richtung Brüssel, sondem um die Erfassung und aus Sicht der involvierten Akteure auch um die dauerhafte Gestaltung der wechselseitigen Beziehungen zwischen der subnationalen und der supranationalen Ebene.

Wie kann nun diese subnationale Mobilisierung greifbar, das heißt vergleichend messbar gemacht werden? In der Mobilisierungsliteratur wurde bisher hauptsächlich untersucht, welche Einflusskanäle und Partizipationsmöglichkeiten subnationalen Entitäten zur Verfügung stehen und wie diese von den Regionen wahrgenommen werden.

* Dieser Artikel entstand im Rahmen des DFG-Forschungsprojektes „Erklärung von GovernancePräferenzen subnationaler Exekutiveliten im Kontext differenzierter subnationaler Mobilisierung:.

1 Cini, Michelle 2003: European Union Politics, Oxford: Oxford University Press.

2 Hooghe, Liesbet 1995: Subnational Mobilisation in the European Union, in: West European Politics, Jg. 18, Nr, 3, S. 175-198; Jeffery, Charlie 2000: Sub-National Mobilization and European Integration: Does it Make Any Difference?, in: Journal of Common Market Studies, Jg. 38, Nr. 1, S. 1-23. 
Dabei haben die einschlägigen Studien im Wesentlichen sechs Kanäle der Repräsentation $^{3}$ identifiziert und die regionalen Aktivitäten in Bezug auf die Nutzung dieser Mitwirkungsmöglichkeiten untersucht. ${ }^{4}$ Wie sich jedoch die subnationalen Gebietskörperschaften in den neuen mittel- und osteuropäischen Mitgliedstaaten in Bezug auf die EU positionieren und sich in der europäischen Politikgestaltung engagieren, ist bislang kaum in empirischen Studien erforscht worden. ${ }^{5}$ Folglich lassen sich bisher kaum Aussagen über das subnationale Mobilisierungspotential der Europäischen Union in den neuen Mitgliedstaaten treffen.

Eine Möglichkeit, Zugriff auf diese Forschungsfrage zu erhalten, ist es, sich zunächst einmal auf die so genannte "attitudinal dimension" ${ }^{\prime 6}$ der subnationalen Mobilisierung zu konzentrieren. Das heißt, man untersucht vorderhand die Einstellungsmuster der zentralen Akteure - der subnationalen Exekutiveliten - im Politikprozess und analysiert, wie sich diese subnationalen Entscheidungsträger gegenüber Aspekten des politischen Systems der Europäischen Union positionieren. Dabei rücken folgende Fragen in den Mittelpunkt: Welche Erwartungen und Vorstellungen haben subnationale Exekutiveliten in Bezug auf die europäische Politikgestaltung entwickelt? Wie schätzen sie die Qualität bestimmter Governance-Mechanismen ein? Wie bewerten sie konkret die Ausgestaltung des Verhältnisses zwischen Region und Europäischer Union und welche Wünsche hegen sie in diesem Zusammenhang für die Zukunft?

Im Rahmen dieses Artikels werden wir diese Ordnungsvorstellungen und Leitbilder subnationaler Verwaltungseliten am Beispiel Polens und Ungarns untersuchen. ${ }^{8}$. Wir

3 Diese sechs zur Verfügung stehenden Einflusskanäle sind der Ausschuss der Regionen, die Regionalvertretungen in Brüssel, der Ministerrat, transnationale Netzwerke, direkte Kontakte zwischen den Regionen und der Europäischen Kommission sowie die Einflussnahme über die nationalstaatlichen Kanäle, z. B. die Zweite Kammer im Parlament.

4 Hooghe, Liesbet/Marks, Gary 1996: "Europe with the Regions": Channels of Regional Representation in the European Union, in: Publius: The Journal of Federalism, Jg. 26, Nr. 1, S. 73-91; Keating, Michael 1998: The New Regionalism in Western Europe. Territorial Restructuring and Political Change, Cheltenham: Elgar.

5 Ausfuhrlich dazu Scherpereel, John A. 2007: Sub-National Authorities in the EU's Post-Socialist States, in: Journal of European Integration, Jg. 29, Nr, 1, S. $23-46$.

6 Sasse, Gwendolyn/Hughes, James/Gordon, Claire 2006: Subnational Governance in Central and Eastern Europe: Between Transition and Europeanization, in: Sadurski, Wojciech/Czarnota, Adam/Krygier, Martin (Hrsg.): Spreading Democracy and the Rule of Law? The Impact of EU Enlargement on the Rule of Law, Democracy and Constitutionalism in Post-Communist Legal Orders, Dordrecht: Springer, S. 121-147.

7 Bauer, Michael W./Pitschel, Diana 2007: Akteurspräferenzen: Schlüssel zur Erklärung subnationaler Mobilisienung. in: Europäisches Zentrum für Föderalismus-Forschung (Hrsg.): Jahrbuch des Föderalismus. Föderalismus, Subsidiarität und Regionen in Europa, Baden-Baden: Nomos, S. 74-85.

8 Die Daten für diesen Artikel stammen aus einem DFG-Forschungsprojekt, das die GovemancePräferenzen subnationaler Exekutiveliten in Bezug auf die allgemeine Architektur der EU und die Ausgestaltung des Verhaltnisses zwischen den Regionen und der EU in fün EU-Mitgliedstaaten (Polen, Ungarn, Frankreich, Spanien und Deutschland) untersucht und das Auftreten bestimmter Präferenzmuster systematisch zu erklären versucht. Diese Staaten wurden ausgewählt, um möglichst unterschiedliche Typen subnationaler Entitäten (politisch-eigenständige mit weitreichenden Kompetenzen vs. administrativ-statistische mit einem begrenzten Spektnum an Kompetenzen, alte vs, neue EU- 
haben den Artikel folgendermaßen aufgebaut: Im ersten Abschnitt wird ein Überblick über die subnationalen Strukturen in beiden Untersuchungsländern gegeben. Daran anschließend setzen wir uns mit der Fragestellung auseinander, inwieweit Präferenzen als Indikator für subnationale Mobilisierung gelten können und geben einen Einblick in die Struktur unseres Untersuchungssamples. Die Präsentation der empirischen Befunde untergliedert sich in zwei Teile. Zunächst werden die Ergebnisse einiger Teilfragen präsentiert, die Aufschluss darüber geben, wie die EU zum gegenwärtigen Zeitpunkt in den untersuchten Regionen wahrgenommen und bewertet wird. Im zweiten Teilabschnitt gehen wir dann der eigentlichen Forschungsfrage nach, nämlich welche Vorstellungen die subnationalen Verwaltungseliten über die zukünftigen Beziehungen zur EU haben. Im letzten Abschnitt fassen wir die Ergebnisse zusammen und diskutieren Implikationen aus unseren Resultaten für die Praxis.

\section{Die subnationale Ebene in Polen und Ungarn}

Sowohl Polen als auch Ungarn gehören zur Gruppe der mittel- und osteuropäischen Staaten, für die sich relativ frühzeitig Perspektiven für eine Mitgliedschaft in der Europäischen Union ergeben haben. Obwohl beide Staaten nach wie vor als unitarisch gelten, nehmen sie im Hinblick auf die Schaffung und Neuordnung regionaler Strukturen unter den mittel- und osteuropäischen Staaten eine Vorreiterrolle ein. ${ }^{9}$ Beide Staaten haben schon in den $1990 \mathrm{er}$ Jahren weit reichende Reformen auf der regionalen Ebene umgesetzt und Regionalwahlen durchgeführt. 10

In Ungarn sind es die Komitate als direkt unterhalb der nationalen Ebene angesiedelten subnationalen Entitäten mit einem demokratisch gewählten Parlament und einer eigenen Verwaltung, die im Rahmen des Projektes die relevanten Aralyseeinheiten darstellen. Die Komitate verfügen über eine lange Tradition im ungarischen Staatswesen. In ihrem jetzigen territorialen Zuschnitt bestehen die 19 Komitate seit den 1920er Jahren (Vertrag von Trianon). Im Jahr 1990 wurde in Ungarn die während der Zeit der kommunistischen Herrschaft außer Kraft gesetzte Selbstverwaltung per Gesetzesakt wieder eingeführt. ${ }^{11}$ Die in Bezug auf Größe und Einwohnerzahlen mit den Kreisen in Deutschland vergleichbaren Komitate verfügen über relativ wenige originäre Kompe-

Mitgliedstaaten, föderale vs. unitarische nationale politische Systeme) im Sample repräsentiert zu sehen.

9 Axt, Heinz-Jürgen 2006: Regionalisierung wider Willen? Die EU-Regionalpolitik und das „Europa der Regionen", in: Zeitschrift für Politikwissenschaft (ZPol), Jg. 16, Nr. 2, S. 457-485.

10 Brusis, Martin 1999: Re-creating the Regional Level in Central and Eastern Europe: An Analysis of Administrative Reforms in Six Countries, in: von Breska, Eric/Brusis, Martin (Hrsg.): Central and Eastern Europe on the Way into the European Union: Reforms of Regional Administration in Bulgaria, the Czech Republic, Estonia, Hungary, Poland and Slovakia, München: Centrum für angewandte Politikforschung (CAP), S. 1-22.

11 Dieringer, Jürgen 2000: Ungarn - Vom ,demokratischen Zentralismus" zur dezentralisierten Demokratie?, in: Europäisches Zentrum für Foderalismus-Forschung (Hrsg,): Jahrbuch des Föderalismus 2000. Föderalismus, Subsidiarităt und Regionen in Europa, Baden-Baden; Nomos, S. 370-383. 
tenzen. In der Hauptsache sind sie für die Bereitstellung öffentlicher Dienstleistung, z. B. den Betrieb von Krankenhäusern oder die Sozialfürsorge zuständig. Neben der regionalen Selbstverwaltung existieren noch weitere dekonzentrierte Verwaltungsorgane, die eine Reihe von Aufgaben (offentlicher Transport, Umweltschutz, Polizei, Raumordnung, Telekommunikation) wahrnehmen. ${ }^{12}$ Seit geraumer Zeit wird in Ungarn eine Neugliederung der bestehenden Regionalstrukturen diskutiert. Dabei sollen durch Zusammenlegung von jeweils drei Komitaten - ähnlich zur bereits bestehenden NUTS II-Struktur - größere mit den westeuropäischen Regionen vergleichbare subnationale Gebietskörperschaften geschaffen werden. Bislang hat man in dieser Sache allerdings noch keine abschließende Einigung erzielen können.

Polen ist seit der großen Regionalreform 1999 in 16 Woiwodschaften untergliedert. Diese sind unter den subnationalen Entitäten in den neuen Mitgliedstaaten in ihrer Struktur (Bevölkerung, Größe) am ehesten mit den westeuropäischen Regionen vergleichbar. Zwischen der Woiwodschafts- und der lokalen Ebene existiert in Polen zusätzlich noch eine Art Kreisebene (Powiaty). Die Woiwodschaften sind zum einen Körperschaften der Selbstverwaltung, gleichzeitig aber auch Träger der staatlichen Verwaltung und Sitz der dekonzentrierten staatlichen Organe. Der Woiwode fungiert dabei quasi als verlängerter Arm der Nationalregierung und beaufsichtigt die Selbstverwaltungsbehörden auf Gemeinde-, Kreis- und Woiwodschaftsebene. ${ }^{13}$ Die Woiwodschaftsselbstverwaltung ist zuständig für die Gestaltung der Regionalpolitik sowie für das regionale Gesundheitswesen, Kultur, Sport, soziale Fürsorge und Bildung.

\section{Tabelle 1: Subnationale Strukturen in Polen und Ungarn}

\begin{tabular}{|c|c|c|c|}
\hline Shene & 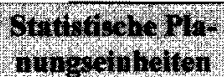 & \multicolumn{2}{|c|}{ 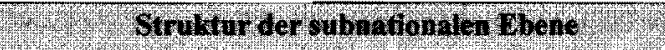 } \\
\hline National & NUTS 0 & \multicolumn{2}{|c|}{ Nationale Regierung } \\
\hline $\begin{array}{l}\text { Subnational } \\
\text { (Region) }\end{array}$ & \multirow[t]{2}{*}{ NUTS $1-3$} & \multirow{2}{*}{$\begin{array}{c}\text { Dezentralisicrte } \\
\text { nationalstaatliche } \\
\text { Verwaltung } \\
\text { Polen, Ungarn }\end{array}$} & $\begin{array}{c}\text { Subnationale Selbstverwaltung } \\
\text { Polen (Woiwodschaft) }\end{array}$ \\
\hline $\begin{array}{l}\text { Subnational } \\
\text { (Kreis) }\end{array}$ & & & $\begin{array}{l}\text { Subnationale Selbstverwaitung } \\
\text { (Kreisebene) } \\
\text { Ungarn (Komitat), Polen (Powiaty) }\end{array}$ \\
\hline Lokal & LAU $1-2$ & \multicolumn{2}{|c|}{ Lokale Selbstverwaltung } \\
\hline
\end{tabular}

NUTS $=$ Nomenclature of Territorial Units for Statistics; LAU = Local Administrative Unit

Quelle: Eigene Darstellung.

12 Bende-Szabó, Gábor 1999: The Intermediate Administrative Level in Hungary, in: von Breska, Eric/Brusis, Martin (Hrsg.): Central and Eastern Europe on the Way into the European Union: Reforms of Regional Administration in Bulgaria, the Czech Republic, Estonia, Hungary, Poland and Slovakia, München: Centrum für angewandte Politikforschung (CAP), S. 23-41.

13 Bondyra, Koysziof/Czachór, Zbigniew 1999: Dezentralisierung und Regionalverwaltung in Polen, in: von Breska, Eric/Brusis, Martin (Hrsg.): Central and Eastern Europe on the Way into the European Union: Reforms of Regional Administration in Bulgaria, the Czech Republic, Estonia, Hungary, Poland and Slovakia, München: Centrum für angewandte Politikforschung (CAP), S. 82-95. 


\section{Präferenzen subnationaler Verwaltungseliten als Indikator für subnationale Mobilisierung}

Die politikwissenschaftliche Forschung hat sich in den vergangenen Jahrzehnten kontrovers mit dem Potential von Präferenzen und Einstellungsmustern zur Erklärung politischen Handelns auseinandergesetzt. Mittlerweile herrscht in der Forschung weitgehender Konsens dahingehend, dass die lange Zeit vorherrschende Skepsis gegenüber Präferenzen in den Sozialwissenschaften bei weitem kein präferenzimmanentes Problem darstellt, sondern auf die unzureichende Spezifizierung des Erklärungsgehaltes von Individualpräferenzen zurückzuführen ist. ${ }^{14}$ Einstellungsmuster sind durchaus ein lohnenswerter Untersuchungsgegenstand und wichtiger Indikator dafür, wie sich Individuen in Bezug auf bestimmte Fragen positionieren und welche Handlungsoptionen sie favorisieren, solange man sich über ihre Funktion und ihre Erklärungsgrenzen im Klaren ist. Präferenzen können somit als eine Art Leitlinie oder Entscheidungshilfe verstanden werden, die dem Individuum einen Handlungsrahmen vorgeben. 15 Einstellungen bleiben situations- und kontextabhängig; allein aus diesem Grund kann aus der Kenntnis der Einstellung eines Individuums nicht ohne weiteres in jeder beliebigen Situation auf dessen tatsächliches Handeln geschlossen werden. Diese Tatsache stellt den Wert der Untersuchung von Präferenzen jedoch keinesfalls in Frage. Denn gerade bei Eliten - so hat eine Reihe von Studien demonstriert - ist ein hohes Maß3 an Übereinstimmung zwischen geäußerten Werten und Einstellungen einer Person und deren tatsächlicher Handlungsweise feststellbar. ${ }^{16}$ Insofern können Akteurspräferenzen durchaus als ein zentraler Baustein zur Erklärung komplexer Phänomene dienen.

Mit einer realistischen Einschätzung des Erklärungsgehalts von Präferenzen - so unser zentrales Argument - kann man auch der Debatte um die subnationale Mobilisierung gerade in den neuen EU-Mitgliedstaaten neue Dynamik verleihen. Zumal sich in der Mobilisierungsforschung selbst Hinweise häufen, dass es neben einer Reihe von institutionellen Faktoren eben entscheidend darauf ankommt, wie sich die exekutiven Eliten vor Ort zur EU positionieren:

,[T]he constitutional framework is not the only factor [...]. The role of elites [...] is also instrumental in the quest for sub-national authorities for greater participation in the EU policy process". 17

14 Hill, Richard I. 1981: Attitudes and Behavior, in: Rosenberg, Morris/Turner, Ralph H. (Hrsg.): Social Psychology: Social Perspectives, New York: Basic Books, S. 347-377; Putnam, Robert D. 1976: The Comparative Study of Political Elites, Englewood Cliffs N. J.: Prentice-Hall.

15 Hooghe, Liesbet 2001: The European Commission and the Integration of Europe: Images of Governance, Cambridge: Cambridge University Press, S. 11.

16 Aberbach, Joel D./Putnam, Robert D./Rockman, Bert A. 1981: Bureaucrats and Politicians in Western Democracies, Cambridge: Harvard University Press, S. 31 ff.

17 Roller, Elisa/Sloat, Amanda 2002: The Impact of Europeanisation on Regional Governance: A Study of Catalonia and Scotland, in: Public Policy and Administration, Jg. 17, Nr. 2, S. 68-86, hier S. 69; siehe auch Jeffery, Charlie $2000(\mathrm{Fn} .2)$, hier S. 15: "The personal authority, interests and 
Dabei nehmen insbesondere die Verwaltungseliten, neben hochrangigen Politikern, eine immer wichtigere Funktion im subnationalen Politikprozess ein. Sie fungieren als eine Art Transmissionsriemen zwischen politischer Gestaltung und Umsetzung. Zudem verfügen sie über vielfältige Möglichkeiten, um ihre Präferenzen, Ordnungsvorstellungen und Überzeugungen aktiv in verschiedenen Phasen des Politikprozesses, z.B. bei der Formulierung von Lösungsvorschlägen für bestimmte politische Probleme, Stellungnahmen oder der Implementation von Gesetzen, aktiv einzubringen. ${ }^{18}$ Gerade in den MOE-Staaten sind es die nationalen, ebenso aber auch die subnationalen und lokalen Verwaltungen, die durch die Ausschöpfung ihrer Gestaltungsmöglichkeiten eine zentrale Rolle bei der Transformation und Demokratisierung der ehemals kommunistischen Systeme spielen. 19

Aufgrund ihrer zentralen Position im Politikprozess stehen die administrativen Eliten auch im Mittelpunkt der vorliegenden Analyse. Das hier untersuchte Teilsample ist folgendermaßen strukturiert. Befragt wurden in beiden Ländern insgesamt 154 Personen in der Funktion eines Abteilungs- oder Referatsleiters. In Ungarn sind 84 Interviews in allen 19 Komitaten, in Polen 70 Befragungen in zwölf Woiwodschaftsselbstverwaltungen ${ }^{20}$ geführt worden. Die Interviews wurden auf der Grundlage eines standardisierten Fragebogens von Muttersprachlern zwischen April und August 2007 durchgeführt.

\section{Empirische Befunde}

\subsection{Einstellungen zur EU und zur subnationalen Politikgestaltung in der $E U$}

Im Rahmen des Surveys wurden einige Fragen gestellt, die Aufschluss darüber geben, wie die europäische Integration, insbesondere die neu erlangte EU-Mitgliedschaft und die von europäischer Seite gemachten Politikangebote, in den Regionen aufgenommen wird. Zunächst wurden die Befragten um ihre Einschätzung bezüglich des Nutzens der EU-Mitgliedschaft für ihr Land und ihre Region gebeten. Insgesamt betrachtet sind die polnischen und die ungarisehen subnationalen Verwaltungseliten der Auffassung, dass sowohl ihr Staat als auch die regionale Ebene von der Mitgliedschaft profitiert haben. Dabei wird der Nutzen für den Staat von der polnischen ebenso wie von der ungarischen

commitment invested by those responsible for leading EU policy in SNAs [subnational authorities] is also an important differentiating variable."

18 Schnapp, Kai-Uwe 2004: Ministerialbürokratien in Westlichen Demokratien. Opladen: Leske \& Budrich.

19 Goetz, Klans H. Dimitrov, Vesselin/Wollmann, Helmui 2006: Post-Communist Executives and European Governance, in: Schuppert, Gunnar Folke (Hrsg): The Europeanisation of Governance, BadenBaden: Nomos, S. 93-131; Jann. Werner 2004: Entwicklung der Ministerialverwaltung in Mittel- und Osteuropa-organisationstheoretische Zugänge und Hypothesen, in: Benz, Arthur/Siedentopf. Heinrich/Sommermann, Karl-Peter (Hrsg): Institutionenwandel in Regierung und Verwaltung. Festschrift fir Klaus König zum 70. Geburtstag, Berlin: Duncker \& Humblot, S. 593-612.

20 Dolnośląskie, Kujawsko-Pomorskie, Lubelskie, Lubuskie, Małopolskie, Mazowieckie, Opolskie, Podkarpackie, Pomorskie, Śląskie, Świętoktzyskie, Wielkopolskie. 
Teilgruppe als höher eingeschätzt als für die Region. Allerdings fallen die Wertungen der polnischen Befragten in Bezug auf beide Fragen deutlich positiver aus als die ihrer ungarischen Kollegen. Vergleicht man die regionalen Antwortmuster, fallt auf, dass in Polen über die Woiwodschaftsgrenzen hinweg deutlich positive Bewertungen abgegeben wurden, während man in Ungarn teilweise enorme regionale Differenzen feststellen kann. So erkennen beispielsweise die Befragten im Komitat Zala tendenziell kaum einen Nutzen in der EU-Mitgliedschaft ihres Landes, während die Befragten in Csongrád in der Mitgliedschaft Ungarns in der EU einen klaren Gewinn für ihre subnationale Einheit sehen (Abbildung 1).

\section{Abbildung 1: Nutzen der EU Mitgliedschaft für das Land}

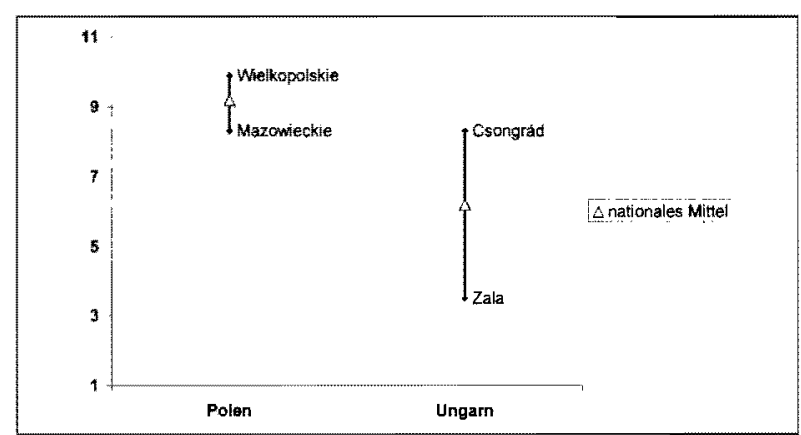

Fragestellung: „Hat ihr Land insgesamt von der EU-Mitgliedschaft profitiert? Die Befragten wurden gebeten. den Nutzen der EU-Mitgliedschaft für ihre Region zu bewerten. Die vorgegebene Antwortskala reicht von 1 (,,kein Nutzen") bis 11 (,großer Nutzen"). Abgebildet sind die Regionen mit den kleinsten und größten Durchschnittswerten sowie das arithmetische Mittel aller Befragten nach Nation.

Quelle: Eigene Darstellung.

\section{Abbildung 2: Nutzen der EU Mitgliedschaft für die Region}

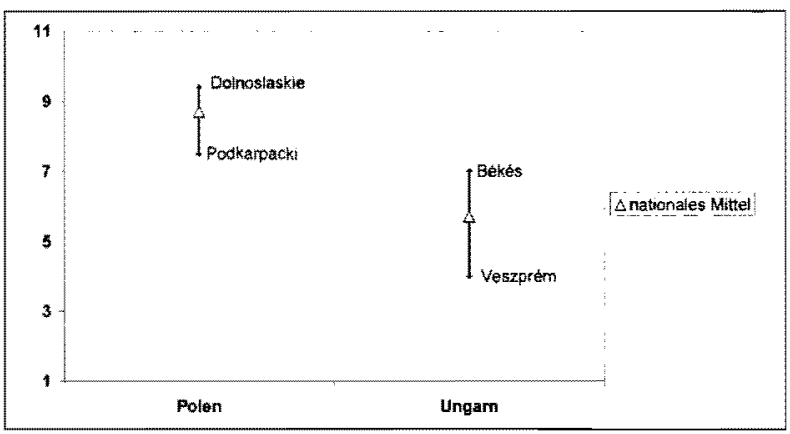

Fragestellung: „Wie bewerten Sie die EU-Mitgliedschaft konkret für thre Region: Hat Ihre Region von der Mitgliedschaft profitiert?" Die Antwortskala reicht von 1 (,,keine Nutzen“) bis 11 (,großer Nutzen“). Abgebildet sind die Regionen mit den kleinsten und größten Durchschnittswerten sowie das arithmetische Mittel aller Befragten nach Nation.

Quelle: Eigene Darstellung. 
Fragt man nach den Möglichkeiten der Regionen, Entscheidungen auf EU-Ebene zu beeinflussen, zeichnet sich - schon allein dadurch, dass die Regionen in beiden Staaten nur über ein begrenztes Spektrum an Kompetenzen verfügen - ein eher gemischtes Bild ab. Während die polnischen Befragten im Durchschnitt eine neutrale Bewertung abgeben (,weder positiv noch negativ $\left.{ }^{64}\right)$, schätzen die Ungarn in der Tendenz ihre Komitate als wenig erfolgreich bei der Beeinflussung der EU-Politik ein. Auch bei dieser Frage offenbaren sich - in diesem Fall in beiden Ländern - große regionale Bewertungsunterschiede. Die Befragten der Woiwodschaft Pomorskie sehen kaum einen Erfolg in Bezug auf regionale Bemühungen, wohingegen die Einschätzung der Interviewten in Podkarpackie deutlich positiv ausfällt. Ähnlich ist die Situation in Ungarn, wo die Bewertungen zwischen ,überhaupt nicht erfolgreich" im Komitat Veszprém und „weder noch" im Komitat Szabolcs-Szatmár variieren (Abbildung 3).

\section{Abbildung 3: Erfolg der Region bei Einflussnahme auf EU-Ebene}

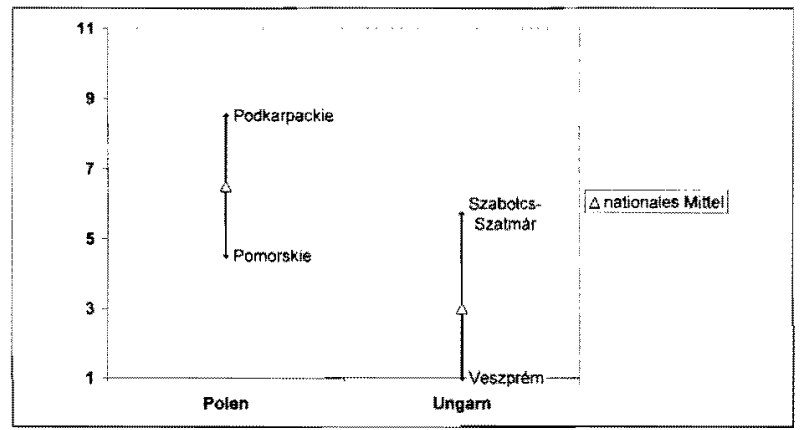

Fragestellung: „Wie schätzen Sie insgesamt den Erfolg threr Region ein, Entscheidungen auf der EUEbene zu beeinflussen?" Die Antwortskala reicht von 1 (,kein Erfolg") bis 11 (,großer Erfolg"). Abgebildet sind die Regionen mit den kleinsten und größten Durchschnittswerten sowic das arithmetische Mittel aller Befragten nach Nation.

Quelle: Eigene Darstellung.

Aufschlussreich sind auch die Antwortmuster in Bezug auf eine Frage nach der Bedeutung des Ausschusses der Regionen (AdR) im direkten Arbeitsbereich der befragten Verwaltungseliten. Die Antworten auf diese Frage variieren in beiden Ländern zwischen der neutralen Kategorie (,,kein Effekt") und dem positiven Pol (.,positiver Effekt"). Erstaunlich ist jedoch, dass in beiden Ländern mehr als die Hälfte der Befragten angibt, dass der AdR in ihrem persönlichen Arbeitsumfeld keine Rolle spielt. In Ungarn sind es sogar knapp 80\% der Befragten, die in ihrem Tätigkeitsfeld nicht mit der Arbeit des AdR in Berührung kommen (Abbildung 4). 


\section{Abbildung 4: Einfluss des AdR im eigenen Arbeitsbereich}

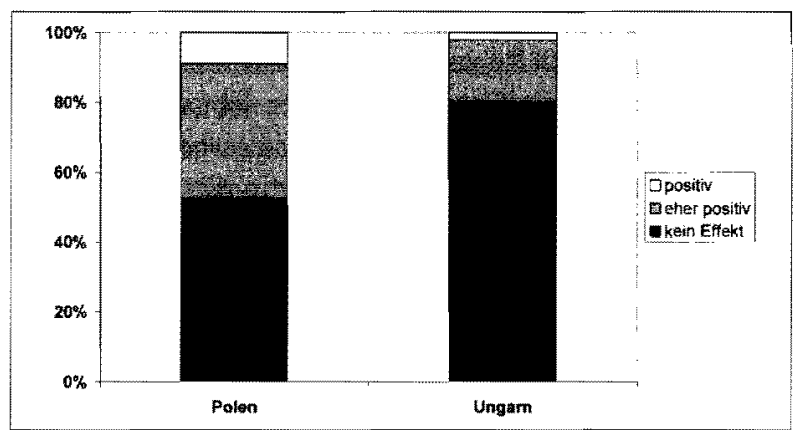

Fragestellung: „Der Ausschuss der Regionen der EU - kurz ,AdR' - besteht aus Vertretern der regionalen und lokalen Gebietskörperschaften aller Mitgliedstaaten und berăt die EU-Kommission, den Ministerrat und das Europåische Parlament. Wie wirkt sich die Arbeit des AdR in Ihrem Arbeitsbereich aus?" Für die drei mogglichen Antwortkategorien wurden jeweils die nationalen Prozentwerte dargestellt.

Quelle; Eigene Darstellung.

\subsection{Leitbilder subnationaler Teilhabe an der europäischen Politikgestaltung}

In einem weiteren Fragenkomplex haben wir die Präferenzen der subnationalen Verwaltungseliten bezüglich der zukünftigen Ausgestaltung des Verhältnisses zwischen der regionalen Ebene und der EU abgefragt.

\section{Abbildung 5: Zustimmung zu partnerschaftlichem Verhältnis zu anderen Institutionen}

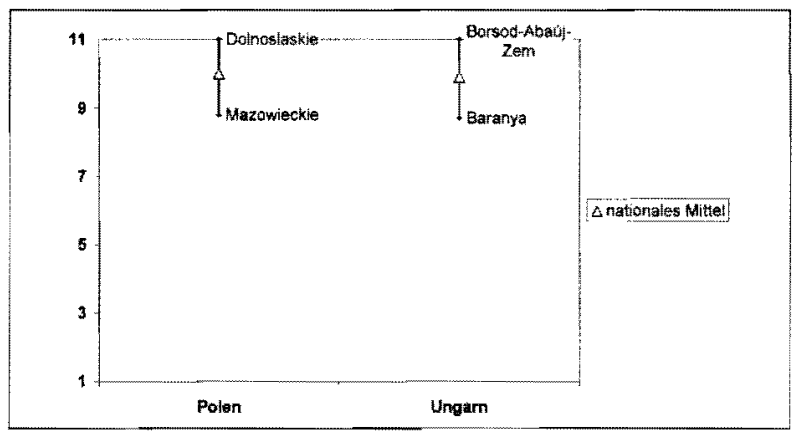

Aussage: „Meine Region sollte ein partnerschaftliches Verhältnis zur EU und dem Nationalstaar haben und in Kooperation mit diesen nach Lösungen für Probleme suchen." Die Antwortskala reicht von 1 (,keine Zustimmung") bis 11 („grole Zustimmung"). Angegeben sind die Regionen mit den kleinsten und größten Durchschnittswerten sowie das arithmetische Mittel aller Befragten nach Nation.

Quelle: Eigene Darstellung.

Im Hinblick auf das allgemeine Verhältnis zur EU und zum Nationalstaat, findet man unter den Befragten in beiden Ländern eine breite Übereinstimmung dahingehend, dass die eigene Region ein partnerschaftliches Verhältnis sowohl zur EU als auch zum Nati- 
onalstaat pflegt und gemeinsam mit beiden nach Lösungen für Probleme suchen soll. Auch zwischen den Komitaten und Woiwodschaften sind in Bezug auf diese Fragestellung im Mittel keine signifikanten Bewertungsunterschiede feststellbar.

Um eine Einschätzung über die Vorstellungen der subnationalen Verwalter im Hinblick auf die Ausgestaltung des Subsidiaritätsprinzips zu bekommen, wurden diese um ihre Meinung zur Einbindung regionaler Parlamente in die Strukturen eines Frühwarnsystems gebeten. Diese Option wurde prominent im EU-Verfassungskonvent diskutiert. $^{21}$ Die nationalen Durchschnittswerte zeigen, dass die Befragten in beiden Staaten die Integration ihrer Regionalparlamente in ein solches Frühwarnsystem durchaus befürworten. Jedoch sind bei dieser Frage deutliche regionale Bewertungsunterschiede festzustellen. In beiden Staaten gibt es Regionen, in denen sich die Exekutiveliten indifferent positionieren (Świętokrzyskie in Polen, Zala in Ungarn), während andere diesem Vorschlag äußerst positiv gegenüberstehen (Kujawsko-Pomorskie in Polen sowie Csongrád in Ungarn).

\section{Abbildung 6: Einbindung der Region in ein Frühwarnsystem}

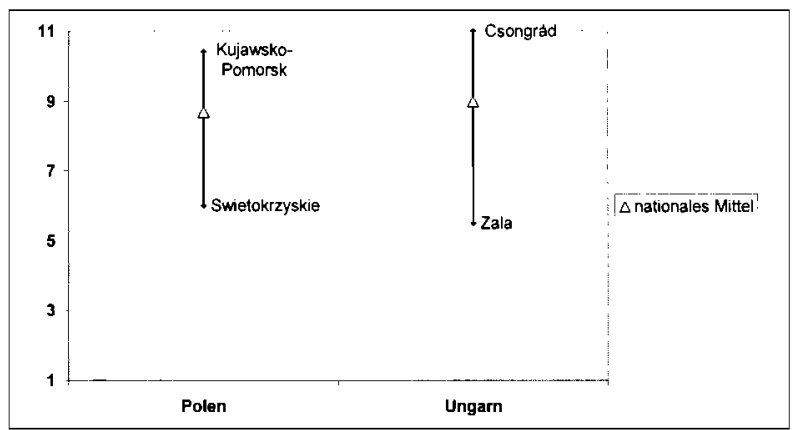

Fragestellung: „Seit Jahren wird diskutiert, in der EU ein so genanntes Frühwarnsystem einzurichten. Dieses ,Frühwarnsystem' sieht vor, dass mitgliedstaatliche Parlamente frühzeitig über Gesetzesinitiativen der EU-Kommission informiert werden und Stellungnahmen abgeben können, wenn sie das Prinzip der Subsidiarität bedroht sehen. Was halten Sie von der Einbindung Ihres regionalen Parlamentes in die nationalen Strukturen eines solchen Frühwarnsystems?" Die Antwortskala reicht von 1 (,keine Befürwortung") bis 11 (,starke Befürwortung“). Abgebildet sind die Regionen mit den kleinsten und größten Durchschnittswerten sowie das arithmetische Mittel aller Befragten nach Nation.

Quelle: Eigene Darstellung.

Auch in Bezug auf die Modalitäten der Kompetenzübertragung auf die EU-Ebene haben die subnationalen Verwaltungseliten in beiden Untersuchungsländern eindeutige Signale gegeben. Die Befragten wurden gebeten, in Bezug auf folgende Aussage Position zu

21 Bauer, Michael W. 2004: Erweiterungsdynamik? Das Europa der Regionen, in: Osteuropa. Zeitschrift für Gegenwartsfragen des Ostens, Jg. 54, Nr. 5-6, S. 160-179; Bourne, Angela 2006: Bringing Europe Closer to the Citizen? Regions, Stateless Nations and the European Convention, in: Regional and Federal Studies, Jg. 16, Nr. 1, S. 1-19; Jeffery, Charlie 2004: Regions and the Constitution for Europe: German and British Impacts, in: German Politics, Jg. 13, Nr. 4, S. 605-624. 
beziehen: „Die EU braucht ein Verfahren, das den Regionen eine Mitentscheidung darüber garantiert, ob und wann Teile ihrer Kompetenzen auf die EU-Ebene übertragen werden." In beiden Staaten findet man im nationalen Durchschnitt hohe Zustimmungswerte. Die meisten Befragten teilen also diese Ansicht. Dieser Trend bestätigt sich auch, wenn man die regionalen Bewertungsmuster in den Blick nimmt. Obwohl eine gewisse Varianz zwischen den Regionen feststellbar ist, wird diese Auffassung in allen im Sample repräsentierten Regionen vertreten.

\section{Abbildung 7: Mitentscheidung bei Kompetenzübertragung}

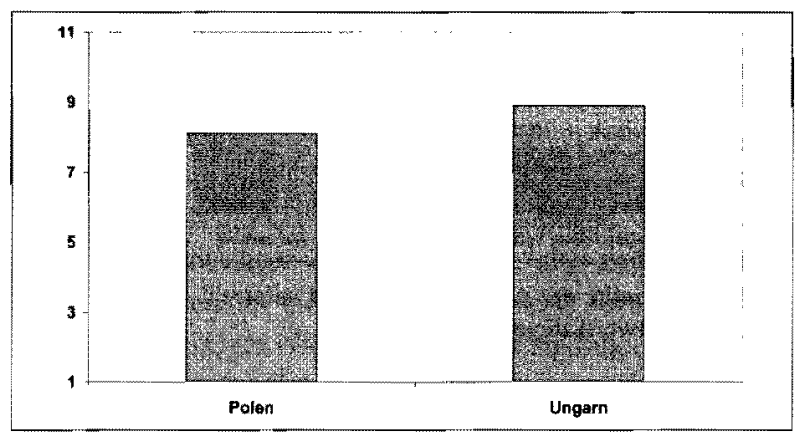

Fragestellung: „Die EU braucht ein Verfahren, das den Regionen eine Mitentscheidung daruber garantiert, ob und wann Teile ihrer Kompetenzen auf die EU-Ebene ubertragen werden." Die Antwortskala reicht von 1 (,stimme uberhaupt nicht $\mathrm{zu}^{\mathrm{k}}$ ) bis 11 (,,stimme $\left.\mathrm{zu}^{\prime \prime}\right)$. Abgebildet sind die nationalen Durchschnittswerte.

Quelle: Eigene Darstellung.

Zudem wurden die befragten Abteilungs- und Referatsleiter in den Woiwodschafts- und Komitatsverwaltungen nach ihren Vorstellungen in Bezug auf die künftige Rolle des Ausschusses der Regionen gefragt. Dabei standen vier Antwortmöglichkeiten zur Auswahl (,abschaffen“; ,soll in jetziger Form weiter bestehen bleiben“; ,soll mehr Rechte bei der Formulierung von Gesetzesvorschlägen haben“; ,soll neben dem Rat und dem Europäischen Parlament eine gleichberechtigte Dritte Kammer werden“). Über $80 \%$ der Befragten haben für Antwortoption drei oder vier votiert und wünschen sich eine Stärkung des AdR (Abbildung 8). Auch über die Regionen hinweg ist dieser Trend eindeutig feststellbar.

Schließlich wurden die Befragten gebeten, zu benennen, welche Ebene (die EU, die nationale Ebene oder die Regionen) gegenwärtig für bestimmte Politikbereiche zuständig ist, und welche nach ihrer Auffassung in Zukunft für diese Politikbereiche verantwortlich sein sollte. Für die hier exemplarisch ausgewählten Bereiche Umwelt, Wirtschaftsförderung und Strukturpolitik sowie Forschung und Technologie lässt sich ein genereller Trend zu einer gewünschten stärkeren Verflechtung der Ebenen ableiten. Während bei der Abfrage des Ist-Zustandes vergleichsweise häufig der Nationalstaat als alleinig verantwortliche Instanz genannt wird, zeigen die Antwortmuster in Bezug auf die gewünschte Kompetenzverteilung, dass sich nach der Einschätzung der regionalen 
Verwalter künftig die Verantwortung nicht mehr ausschließlich bei den Nationalstaaten konzentrieren wird, sondern auch die beiden anderen Ebenen (also EU und Regionen) eine stärkere Gestaltungsfunktion übernehmen sollen (Tabelle 2).

\section{Abbildung 8: Zukünftige Rolle des AdR}

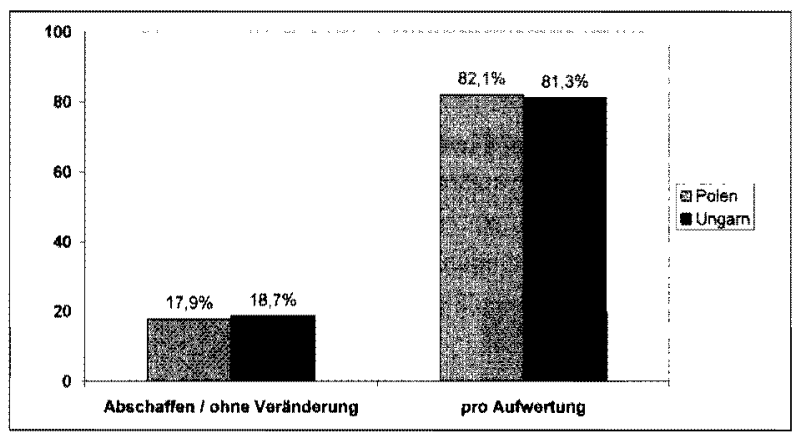

Fragestellung: „Welche Rolle sollte der AdR in der Zukunft spielen“? Abgebildet sind die Prozentwerte für die zusammengefassten Antwortkategorien nach Nation.

Quelle: Eigene Darstellung.

Tabelle 2: Kompetenzallokation zwischen den Governance-Ebenen

\begin{tabular}{|c|c|c|c|c|c|c|c|}
\hline \multirow[t]{2}{*}{ 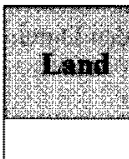 } & \multirow[t]{2}{*}{ Whene } & \multicolumn{2}{|c|}{ (m) Prowent) } & \multicolumn{2}{|c|}{ 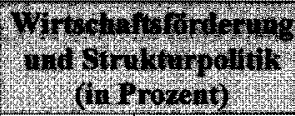 } & \multicolumn{2}{|c|}{ 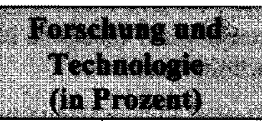 } \\
\hline & & IST & SOLL & IST & SOLL & IST & SOLL \\
\hline \multirow[t]{3}{*}{ Polen } & EU & 41 & 52 & 52 & 50 & 18 & 53 \\
\hline & National & 90 & 62 & 88 & 65 & 93 & 73 \\
\hline & Regional & 37 & 55 & 42 & 68 & 26 & 42 \\
\hline \multirow[t]{3}{*}{ Ungarn } & EU & 34 & 69 & 69 & 48 & 29 & 58 \\
\hline & National & 67 & 23 & 66 & 43 & 73 & 41 \\
\hline & Regional & 2 & 13 & 0 & 16 & 0 & 5 \\
\hline
\end{tabular}

Die Prozentzahlen geben den prozentualen Anteil an den insgesamt gegebenen Antworten pro Land wieder (Mehrfachnennungen möglich).

Fragestellung: „Welche Ebene - die EU, die Nationalstaaten oder die regionale Ebene - ist Ihrer Meinung nach aktuell der Hauptverantwortliche fur das Politikfeld? Welche der drei Ebenen sollte zukunftig der Hauptverantwortliche sein?"*

Quelle: Eigene Darstellung.

\section{Subnationale Mobilisierung in Polen und Ungarn}

Diese deskriptive Auswertung von einschlägigen Einstellungsmuster zeigt - mit der angemessenen Vorsicht bezüglich der Verallgemeinerbarkeit der erhobenen Individualpräferenzen als institutionelle Regionalpräferenzen -, dass die subnationalen Verwaltungseliten 
in Polen und Ungarn insgesamt betrachtet der EU in der Tendenz positiv gegenüberstehen. Wenn man nun die relativ nationalistischen Töne der Warschauer Zentralregierung dagegenhält, deutet das beispielsweise darauf hin, dass diese Politik in den polnischen Regionen eigentlich keinen Rückhalt hat. Allerdings zeigen die Ergebnisse auch, dass es deutliche nationale und regionale Unterschiede im Hinblick darauf gibt, wie das europäische Integrationsprojekt zum gegenwärtigen Zeitpunkt von den Verwaltungsbeamten wahrgenommen und bewertet wird. Das ist insbesondere dort der Fall, wo der Erfolg der EU-Mitgliedschaft für den Nationalstaat anders eingeschätzt wird als der Gewinn für die Region. Dabei wird deutlich, dass manche Regionen äußerst optimistisch gestimmt sind, in anderen hingegen eindeutig eine gewisse Skepsis dominiert. Das bedeutet, dass ,Euro$\mathrm{pa}^{: *}$ eben (noch) nicht in allen neuen Mitgliedstaaten, geschweige denn in allen Regionen, gleichermaßen ,angekommen“ ist bzw. die Partizipationsmöglichkeiten, die die europäische Integration mit sich bringt, noch sehr unterschiedlich bewertet und vermutlich auch genutzt werden. Bemerkenswert ist in diesem Zusammenhang auch, dass gerade auf europäischer Ebene agierende regionale Interessenvertretungen wie der Ausschuss der Regionen kaum von den Exekutiveliten in den Regionen, also von ihren eigentlichen Adressaten, wahrgenommen werden. In diesem Sinne besteht, sowohl auf Seiten der EU als auch auf der der Regionen, einiger Nachholbedarf, insbesondere was die Kommunikation und Information über Politikangebote und deren Nutzung angeht.

Die prospektiv ausgerichteten Fragen, die die Zukunftsvorstellungen und Gestaltungswïnsche der subnationalen Verwaltungseliten in den Blick nehmen, vermitteln hingegen ein überwiegend optimistisches Bild. Die äußerst positiven Reaktionen auf unsere Fragestimuli lassen erkennen, dass die Befragten durchaus noch Potentiale sehen, die Rolle ihrer Regionen im europäischen Politikgestaltungsprozess künftig auszubauen und die dritte Ebene als solche zu stärken. So deuten beispielsweise die Antworten auf die Fragen nach der Integration der regionalen Parlamente in die Strukturen des Frühwarnsystems sowie zur Möglichkeit der Entsendung eines regionalen Vertreters in den Ministerrat darauf hin, dass die subnationalen Entitäten - auch wenn ihre Kapazitäten gegenwärtig begrenzt sind - grundsätzlich bereit sind und sich auch in der Lage dazu sehen, in Zukunft mehr institutionalisierte Verantwortung und anspruchsvolle Aufgaben im europäischen Mehrebenensystem zu übernehmen. In Bezug auf die Ausgangsfragestellung lässt sich damit festhalten, dass es - wenngleich differenziert - bereits gelungen ist, Verwaltungseliten in den Regionen für die neuen Herausforderungen europäischer Mehrebenenpolitik zu sensibilisieren. Es gibt allerdings noch ein bislang „ungenutztes" Mobilisierungspotential in den untersuchten Regionen, das aus einem Personenkreis besteht, der, obwohl er die gegenwärtigen Möglichkeiten als begrenzt einschätzt, sich künftig durchaus einiges von der europäischen Integration für seine subnationale Einheit erwartet. Es wird sich also erst noch zeigen, wie sich das vorhandene Mobilisierungspotential mittelfristig entfalten wird und wie sich die bislang relativ passiven subnationalen Gebietskörperschaften in Mittel- und Osteuropa in den europäischen Politikprozess einbringen werden. Aus integrationsfreundlicher Sicht ist die EU allerdings gut beraten, ihre subnationale Klientel in Osteuropa mit entsprechenden Anreizen, seien diese sachpolitischer oder supranationalkonstitutioneller Art, für eine verstärkte europapolitische Zusammenarbeit zu gewinnen. 